\title{
LES EXPRESSIONS DÉICTIQUES AI ET PRAI. REGISTRE COLLOQUIAL, APPROXIMATION ET RESPONSABILISATION ÉNONCIATIVE
}

\author{
MARIA ALDINA MARQUES ${ }^{1}$
}

\begin{abstract}
Deictic expressions aí and praí. Colloquial register, hedging and enunciative responsibility. The aim of this paper is to analyse the semanticpragmatic features of Portuguese expressions aí and praí in context. Our study of these linguistic structures starts with the observation of their occurrences in a corpus of spontaneous spoken language that can be considered as a rather colloquial register of Braga's speech, consisting of about 80 hours of recordings. The analysis of aí and praí raises complex but interesting questions from the point of view of oral communicative competence, especially with regard to the acquisition of Portuguese as a foreign language. Following an enunciative-pragmatic approach, we highlight the multifunctionality of these deictic expressions. In fact, besides deictic values, aí and praí are also linguistic mechanisms of hedging, signalling in particular the weakening of the enunciative responsibility of the speaker.
\end{abstract}

Keywords: deixis, spatial deictics, hedging, enunciative responsibility, subjectivity.

REZUMAT. Expresiile deictice aí și praí. Registru colocvial, atenuare și responsabilitate enunțiativă. Obiectivul acestei lucrări este să analizeze în context caracteristicile semantico-pragmatice ale expresiilor portugheze aí and praí. Studierea acestor structuri lingvistice începe cu observarea ocurențelor într-un corpus de limbă vorbită, care poate fi considerat mai degrabă registru colocvial din Braga și care conține aproximativ 80 de ore de înregistrări. Analiza expresiilor aí și praí pune probleme interesante din punctul de vedere al competenței de comunicare orală, în special în ceea ce privește învățarea portughezei ca limbă străină. Adoptând o abordare enunțiativă și pragmatică, subliniem multifuncționalitatea acestor expresii

1 Maria-Aldina MARQUES est professeure à l'Institut de Lettres et Sciences Humaines de l'Université du Minho au Portugal. Elle a soutenu sa thèse de doctorat sur l'organisation énonciative du discours parlementaire portugais. Ses domaines de recherche privilégiés dans l'analyse du discours sont les discours politiques, les discours des médias et les discours de l'oral quotidien. E-mail: mamarques@ilch.uminho.pt 
deictice. De fapt, pe lângă valorile deictice, aí și praí sunt și mecanisme lingvistice de atenuare, ce semnalizează în special o responsabilitate enunțiativă mai slabă a vorbitorului.

Cuvinte cheie: deixis, deixis spațial, atenuare, responsabilitate enunțiativă, subiectiviate.

\section{Introduction. Quelques remarques préliminaires}

Le but de notre travail est d'analyser les caractéristiques sémanticopragmatiques des expressions portugaises aí [e'i], et praí [pra'i], en contexte oral spontané, voire colloquial2 ${ }^{2}$ Praí est la façon habituelle dont on prononce en portugais la séquence para aí. On y voit quelques-unes des caractéristiques du portugais oral spontané: l'élision de voyelles en syllabe pré-tonique (para > p'ra) et la crase des deux [e] (p'ra aí > praí). Praí est une expression figée, dérivée de l'amalgame de la préposition PARA [pour; à, par] et de l'adverbe de lieu $A I ́$ [ $\approx$ y; ici, là], que nous allons donc traiter en bloc.

\section{Cadrage théorique et méthodologique}

Nous avons adopté une démarche énonciative-pragmatique pour analyser les usages des constructions linguistiques aí et praí dans le cadre des catégories déictiques, pour parvenir à d'autres usages discursifs marqués par valeurs approximatifs ${ }^{3}$.

La deixis a été abordée par une multitude de chercheurs travaillant dans des courants linguistiques divers, mais pour ancrer notre analyse nous avons choisi, tout en particulier, les théories de Benveniste (1974) sur l'énonciation, reprises et redéfinies par Ducrot (1984) et Rabatel (2010), entre autres, notamment en ce qui concerne la distinction locuteur - énonciateur. Ces approches mettent en relief le caractère foncièrement dialogique de l'organisation énonciative du texte/discours. Nous considérons en outre que l'organisation énonciative détermine la référenciation et l'argumentation en discours (Mondada \& Dubois, 1995; Rabatel, 2005; Amossy, 2000).

En ce qui concerne l'approximation et ses effets de sens, la définition de hedging, présentée par Fraser (2010), est notre point de depart: «... a rhetorical

${ }^{2}$ L'interview sociolinguistique semi-dirigée, qui constitue notre corpus, est hétérogène quant au registre de langue utilisée.

3 ...la fonction approximative a tendance à s'ancrer dans la signification de certaines expressions conventionnalisées qui rendent floue la signification de l'élément qu'ils modifient (Mihatsch, 2009: 101). 
strategy that attenuates either the full semantic value of a particular expression, as in He's sort of nice, or the full force of a speech act, as in I must ask you to stop doing that ». Ces valeurs sont présentes dans les usages d'aí et praí étudiés.

Notre réflexion sur ces structures linguistiques part de l'observation de leurs occurrences dans un corpus de langue parlée spontanée (de Braga), que l'on peut considérer comme un registre plutôt colloquial (Marques, 2015), constitué de 80 heures environ d'enregistrements ${ }^{4}$.

Notre but, nous l'avons déjà signalé, est d'analyser les valeurs sémanticopragmatiques de aí et praí en contexte. Nous posons comme hypothèses de travail que le positionnement du locuteur dans la relation à son allocutaire et au contenu de l'énoncé détermine la construction de la spatialité. De plus, l'éloignement spatial est une valeur nucléaire de aí, d'où dérivent d'autres valeurs discursives, évaluatifs et approximatifs, de aí et praí.

Finalement, praí et aí sont des mécanismes linguistiques de l'approximation signalant, notamment, l'atténuation de la responsabilité énonciative du locuteur.

Ce sont des usages stratégiques, marquant l'adéquation du locuteur au dit, au dire et à son interlocuteur; il s'agit de la négociation explicite du sens, qui sous-tend tout processus de référenciation.

\section{Déixis spatiale - État de l’art}

\subsection{La perspective traditionnelle}

En Portugais, les déictiques de lieu sont une catégorie complexe, organisée en deux séries, complémentaires, l'une ternaire et l'autre binaire, respectivement Aqui, aí, ali (acolá) et cá, lá5.

La grammaire traditionnelle a limité l'analyse de ces formes à la valeur sémantique de locatif, d'où la catégorisation comme adverbes de lieu, dont elle ne signale qu'une liste unique (Cunha \& Cintra, 1984; Vasquez Cuesta \& Luz, 1971).

\subsection{Fonction déictique d'une catégorie énonciative}

La pragmatique et, en particulier, la linguistique de l'énonciation ont redessiné les approches et les analyses, en considérant la catégorie dans le cadre de l'énonciation comme un de ses paramètres.

Ainsi, l'adverbe aí fait partie d'une catégorie à 3 pôles, que l'on rapproche de la catégorie des pronoms personnels (eu, tu, ele/ela [je, tu,

\footnotetext{
4 Voir en annexe les conventions de transcription.

5 Il y a une troisième série, constitué de deux éléments além, aquém, ayant des usages plus restreints et des spécificités que nous n'aborderons pas.
} 
il/elle]) ou des personnes verbales $\left(1 \mathrm{e}, 2^{\mathrm{e}}\right.$ et $3^{\mathrm{e}}$ personnes verbales), respectivement: aqui [ici, près de moi], aí [ici/là, près de toi] et ali [là, ni près de moi ni près de toi]. On y reconnait, donc, un centre déictique délimité par les trois paramètres moi, ici, maintenant 6 .

Cette catégorisation, tournant autour du locuteur - le " deitic center » (Lyons, 1995:304) -, est réductrice et ne rend pas compte de la complexité des usages en contexte ${ }^{7}$. Il faut prendre en considération la relation entre le locuteur et l'allocutaire qui permet de construire un espace commun premier, délimité en fonction de leur rôle d'interlocuteurs; c'est l'espace abstrait des participants à l'interaction, un espace partagé. À une conception tripartite homogène et homologue des trois personnes verbales, on substitue donc une autre, ancrée sur l'espace commun de l'énonciation par opposition à un espace extérieur aux relations interactionnelles ${ }^{8}$. À l'espace énonciatif construit par le seul locuteur en fonction de soi-même, il faut superposer une autre organisation spatiale manifestant un espace commun interlocutif, bien que cet espace « consensuel de base ", que les séries à deux termes nécessairement construisent et le portugais prévoit aussi dans la série cá - lá, n'efface pas la distinction entre l'espace du moi et celui du toi. Le schéma ci-dessous illustre cet agencement spatial différencié:

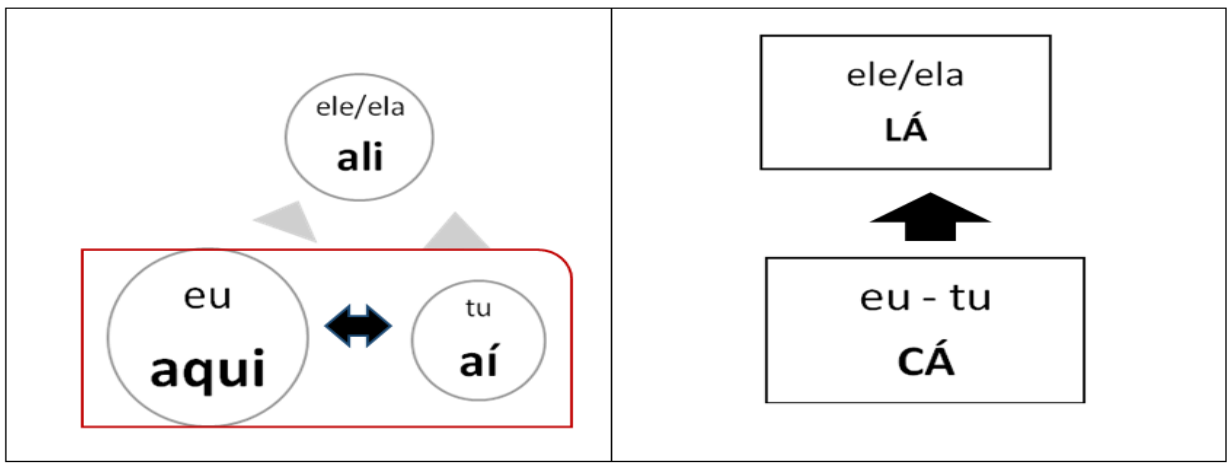

Schéma 1. Agencement spatial

\subsubsection{Fonction déictique exophorique}

\subsubsection{Proximité, indéxicalité, visibilité et accessibilité}

Étant donné les objectifs de notre recherche, nous n'allons considérer que certains usages « exophoriques ${ }^{9}{ }^{\text {de cette série. }}$

${ }^{6}$ Sur la déixis en portugais, voir Fonseca $(1992,1995,1996)$.

7 Voir Kleiber (1986:12) pour une thèse critique de l'égocentrisme.

${ }^{8}$ Teixeira (2005) signale l'importance de cette réorientation de la catégorisation.

${ }_{9}$ Pour la classification des valeurs exophoriques et endophoriques des catégories déictiques, voir Halliday et Hasan (1976). 
L'organisation interne de la série met en relief une relation (graduelle) de proximité10 à des points de repère, mis en scène par un locuteur-énonciateur responsable du point de vue présenté, en relation avec un allocutaire-destinataire qui doit interpréter l'énoncé. Le centre déictique dont parle Lyons (1995: 310) est, dans ce sens, un centre partagé.

Cette proximité est, dans un premier temps, en lien étroit avec l'indéxicalité, au sens le plus stricte, d'activité d'ostension proposé par Buhler (1934) ou « gestural reference » (Lyons, 1995: 303), ancrée sur une notion de spatialité « visible » qui gouverne la série. En fait, on peut montrer du doigt la localisation spatiale d'un objet.

Ces caractéristiques ostensives mettent en relief la visibilité de l'objet localisé. Toutefois, les occurrences enregistrées montrent qu'il s'agit d'une délimitation spatiale esquissée en fonction d'un point de vue. En d'autres mots, ce sont des configurations spatiales de proximité qui découlent d'un positionnement évaluatif du locuteur et que celui-ci négocie avec son allocutaire. Ce n'est pas une délimitation d'espaces géographiques mais d'espaces gouvernés par une perception nettement subjective, qui suppose notamment des connaissances partagées et l'adhésion du locuteur au point de vue mis en scène ${ }^{11}$. Il faut souligner que dans cette négociation, qui est foncièrement argumentative (Amossy, 2000), l'objet est montré en tant que cognitivement accessible à l'allocutaire.

C'est pourquoi, dans une situation quelconque, on peut créer des délimitations spatiales à ampleurs diversifiées, en fonction du choix du locuteur et des visées argumentatives qu'il poursuit: "Aqui/aí/ali, au Portugal », «aqui/aí/ali, à Braga », «aqui/aí/ali sur cette table ».

Aqui (et aussi aí, ali) peut faire référence à des espaces diversifiés, délimités en premier lieu par la visée du locuteur qui surplombe la dimension de l'espace physique où se trouvent les interlocuteurs. C'est ce qui arrive dans une situation d'énonciation où le locuteur peut choisir entre ces deux énoncés «Vamos aqui para a sala /allons dans cette salle-ci » et «Vamos ali para a sala (allons dans cette salle-là) », parce que la proximité de lieu est tout à fait subjective (et argumentative).

Dans ce décalage subjectif par rapport à l'espace de l'énonciation, c'est particulièrement intéressant l'usage de ali pour marquer un espace de mémoire (Buhler, 1934; Fonseca, 1996; Barbéris, 1998), récupérable seulement à partir

10 Je renvoie à Lyons (1995: 310) pour une définition de ce terme, qui est néanmoins un peu restrictive: « ...the notion of spatio-temporal proximity to the deictic center (...) this and here refer to entities and places that are located in the place that contains de speaker (...) that is what proximity means when it is used, technically, in discussions of deixis ».

11 C'est aussi la perspective de Barbéris (1998: 28): il s'agit plutôt d'un point de vue subjectif que d'une localisation effective des interlocuteurs. 
des connaissances partagées des interlocuteurs ( 0 palácio fica ali na rua do Raio. » /Le palais est là, rue du Raio) ${ }^{12}$ ou, pour mieux dire, des connaissances données comme partagées. L'exemple (1) ci-dessous présuppose un partage de connaissances qui en fait n'existe pas; l'allocutaire (l'intervieweur, dans ce cas) ne connait pas et ne voit pas l'immeuble dont il est question, mais, en utilisant «ali » comme localisateur déterminant un objet spécifique, le locuteur rend l'objet discursif accessible à son interlocuteur. Malgré tout, « ça marche » et le locuteur réussi à communiquer:

(1) • Um gajo vai ali para a sede, também bebe umas cervejotas. • Bebe cinco cervejas, $(12 \mathrm{H} 2 \mathrm{~A})^{13}$

[• • un mec $y$ va au siège [du groupe sportif], il boit aussi des bières $\bullet \bullet$ il boit cinq bières]

Cette caractéristique du déictique est aussi présente dans d'autres usages, d'intensification de l'évaluation. Le lien qu'il établit avec le «je» énonciateur entérine cette interprétation:

(2) Ainda lavrei muito com o arado atrás dos bois [...] a virar a terra, ((hesitação)) ali a fazer músculo para segurar, para a terra virar, ficar ali direitinha. (25H3B)

[j'ai encore labouré à la charrue derrière les bœufs [...] à ouvrir le sillon ((hesitation)) là en faisant force pour maintenir, pour ouvrir le sillon là bien droit]

\subsubsection{Fonction déictique temporelle}

La valeur de proximité règle aussi les usages temporels, ou mieux spatio-temporels, un usage exophorique, de aqui, aí, ali ayant comme point de repère le temps-espace de l'énonciation ${ }^{14}$. On a, entre autres, ces possibilités (a) « Daqui a pouco vou para casa. [je m'en vais, dans peu de temps]",

12 Barbéris (1998: 32) conclut pour le français que: «Sans doute convient-il de souligner l'inadéquation des modèles descriptifs de la déixis reposant exclusivement sur la localisation du réfèrent (localisation dans le contexte /dans le cotexte) (Kleiber 1991). Attribuer une valeur mémorielle aux formes en L-, dont fait partie l'adverbe là, permet d'y voir plus clair, par opposition aux formes en C-, non mémorielles, où se situent les démonstratifs (adjectifs, pronoms), ainsi que l'adverbe ici. La désignation en là montrerait donc un objet préconstruit, déjà connu, tandis qu'ici procéderait à un découpage spatial $a b$ initio, au moment de son occurrence ».

13 J'ai traduit tous les exemples. C'est une traduction approximative; le seul souci a été de préserver les valeurs des unités analysées.

14 Nous n'avons pas pris en compte les usages ayant un point de repère autre que le temps de l'énonciation: «Ele saiu. Dali a minutos começou a chover. [Il est sorti. Quelques minutes plus tard, il a commencé à pleuvoir.] ». « Dali a minutos » montre l'éloignement temporel face à l'évènement «il est sorti »). 
marquant l'éloignement par rapport à l'espace-temps des interlocuteurs; (b) « Vem aí chuva. [Il va pleuvoir] ", qui établit l'approximation à l'espace-temps des interlocuteurs.

La surdétermination de la localisation déictique spatiale et temporelle par le positionnement énonciatif du locuteur-énonciateur configure ces localisations. En (3), la même période est représentée soit par aqui soit par ali. Plutôt que d'y avoir des usages temporels, aqui e ali sont surtout évaluatifs en confluence avec une certaine imprécision factuelle; le locuteur raconte quelque chose de désagréable (des vols) qui a eu lieu il y a peu de temps, pendant une période vaguement délimitée:

(3) É para ver a coisa que não vai há uma década que isso era impossível era • • era impensável. • • E hoje já já se vê. • • Pronto, não é que seja assim, mas houve uma altura mais aqui há um ano atrás, $\bullet \bullet$ houve ali um período/ cerca de duas semanas, aquilo era/ os assaltos eram constantes. $(18 \mathrm{H} 2 \mathrm{C})$

[...il y a un an, $\bullet$ on a eu une période/ deux semaines environ, ça était/ les vols étaient constants]

\section{Aí et praí dans le corpus du parler de Braga}

\subsection{Aí, déictique exophorique et endophorique}

Le fonctionnement de aí a fait l'objet de descriptions assez nombreuses soit comme adverbe de lieu soit en tant que déictique et anaphorique (Fonseca, 1992, 1995, 1996; Raposo et al., 2013; Pereira, 2009; Teixeira, 2005).

En tant que déictique exophorique, aí présente les valeurs nucléaires de la série, c'est-à-dire, de localisation spatiale (4), dans l'espace de allocutaire, et temporelle (5), signalant la proximité temporelle relativement au temps de l'énonciation:

(4) ((risos)) • • E tocavam à campainha: ((hesitação)) - Sim, faz favor. - Ó menina Laura, está aí o paizinho? • • ((hesitação)) - Eu vou ver. Se faz favor, de entrar. (88M4D)

[((rires)). • et on sonnait à la porte: ((hésitation)) - Oui, s'il vous plaît. - Mlle Laura, votre papa est là?.•• ((hésitation)) - Je vais voir. Entrez, s'il vous plait]

(5) E: • • A família. • • Hum, hum. • • Olha, e em relação às touradas? • • Vem vem aí o verão, é assim uma coisa • - que se costuma ver muito. (ENTRE:)

[• - La famille $\bullet \bullet$ hum, hum. $\bullet$ Tiens et les courses de taureaux? • L'été est presque là, c'est une chose•• que l'on voit beaucoup] 
Dans notre corpus, la valeur exophorique de aí est associée aux circonstances de l'interaction (5) mais surtout au discours rapporté (4), étant donné la fréquence des récits conversationnels.

La localisation spatiale-temporelle peut être aussi endophorique, c'est-à-dire, textuelle ${ }^{15}$. Aí est un marqueur de l'organisation discursive, à valeur anaphorique (remplaçable par dans ce cas-là; à ce moment-là) (7); mais d'autres valeurs peuvent s'y agréger, c'est le cas de (6) dont la valeur anaphorique s'accompagne d'une valeur de présentatif intégré à une énumération à valeur argumentative:

(6) E depois, eu não gosto de me ver metida no meio de tanta gente. Aí está outro problema. (07H1D)

[Et en outre, je n'aime pas dans la foule. Et voilá un autre problème.]

(7) I: Se for num serviço de urgência, como é óbvio, • • aí não se faz $\bullet$ metade do que se devia fazer, mas $\bullet \bullet$ se for num serviço normal, basicamente é isso. (90M4D)

[s'il s'agit d'un service d'urgence, évidemment, • • Dans ce cas-là on ne fait ni la moitié de ce qu'on devrait faire.]

\subsection{Aí - des usages évaluatifs et approximatifs}

Nous avons vu que la déixis spatiale, que nous sommes en train d'analyser, est déterminée par le point de vue de l'énonciateur, lequel redéfinit les espaces. Cette subjectivité s'impose dans d'autres emplois discursifs à valeurs d'évaluatif (positif ou négatif selon les contextes d'occurrence) et d'approximateur, bien marquées.

En faisant une évaluation négative, aí permet de tracer un premier degré de distanciation du locuteur par rapport au contenu de son énoncé. Il met en scène un énonciateur qui ne dit pas tout, qui ne respecte pas la maxime de la quantité de Grice. Aí devient un marqueur d'évaluation négative. Le locuteur prend ses distances par rapport au contenu de l'énoncé, parce qu'il ne veut pas tout révéler (10), ou parce qu'il en a une opinion négative et veut renforcer son acte de critique (8) et (9) ${ }^{16}$ :

15 L'exemple présenté par Teixeira (2005) « Foi para o Porto e aí/lá viveu », que l'auteur caractérise comme neutre par rapport à l'opposition aí-lá s'étaye à mon avis sur une lecture plus déictique ou plus textuelle de l'événement. Cela met en relief le continuum d'usages déictiques exophoriques et textuels, déterminés par un positionnement énonciatif du locuteur.

${ }^{16}$ La continuation discursive montre des compatibilités qui renforcent ces valeurs contextuelles. La valeur évaluative négative, par exemple: «quantos estádios temos aí agora que não são utilizados? É uma vergonha! [Combien de stades y avons-nous maintenaient qui ne sont pas utilisés? c'est un scandale]». 
(8) Mas não andes aí a espancar que ela não é nenhum molho de palha. (88M4D)

[Mais tu ne dois pas la bâtonner, puisqu'elle n'est pas une gerbe de paille]

(9) Por exemplo, o futebol, quando foi o Euro 2004, quantos estádios temos aí agora do Euro 2004 que não são utilizados? (XXX)

[Par exemple, le football, il y a eu l'Euro 2004, combien de stades y avons-nous maintenaient qui ne sont pas utilisés?]

(10) E não vá muito longe • • que eu faço parte também aí dum, • • pronto, trabalho um bocado no INATEL ((hesitação)) a aprender umas coisas e assim (88M4D)

[Et voyez•• moi je fais partie d'un• • bon, je travaille un peu à INATEL (hésitation)) j'y apprends des trucs]

Quand le locuteur ne veut pas tout dire et il crée une distance subjective, que le cotexte renforce, la non-identification de l'objet de discours, les hésitations, les reformulations, les déterminants indéfinis et d'autres mécanismes de langage vague contribuent tous à la construction de cette ambiance de détachement et de (non-) prise en charge. On y voit une sous-information qui est caractéristique de l'allusion, de la rumeur. C'est ce positionnement énonciatif qui régit l'occurrence suivante:

(11) Olhe, há aí um que vem aí visitar uma tia. (90M4D)

Tenez, il $\mathbf{y}$ en a un qui y vient visiter une tante.]

Mais c'est le contexte à valeur positive ou négative, et l'acte illocutoire notamment, qui détermine l'interprétation de aí comme renforcement et proximité ou écart du locuteur par rapport au contenu et à l'interlocuteur ${ }^{17}$ :

(12) I: Se a claque decide $\bullet$ - destruir um autocarro ou qualquer coisa do género, o clube não, não foi o clube que disse: - Olha, destrói aí.

[Vas-y, détruis tout]

(13) E: Diz-me aí então os teus livros assim preferidos.

[Dis-moi, donc, quels sont tes livres préférés.]

(14) E: Devia ter aí umas pernas com um músculo.

[Vos jambes, elles devraient être bien musclées]

Finalement, les structures de quantification, si fréquentes dans notre corpus, permettent aussi un usage approximatif de aí, véhiculant le sens de « plus ou moins; environ »18:

17 Landone (2012: 307) cite Chodorowska-Pilch (2008: 1359) sur ce genre de fonctionnement: " [...] it is the metacommunicative movement beyond the content of the utterance that produces the efect od distancing or closeness between interlocutors ». 
(15) Depois temperam-se com vinho tinto, alho, pimenta $\bullet \bullet$ e sal. $\bullet \bullet$ E depois deixam-se estar aí dois ou três dias, • • em alguidares de barro (86M4C). [Après on les assaisonne de vin rouge, ail, pimente $\bullet$ et sel. $\bullet \bullet$ Et puis on les met pendant deux ou trois jours, $\bullet$ dans un bol de céramique]

\subsection{Praí - usages, sens et contextes}

Praí a des valeurs qui font partie de ces catégories ci-dessus présentées pour aí. Toutefois, la construction avec la préposition para le spécialise dans certains effets de sens.

En fait, pour déterminer les usages discursifs de cette structure, la valeur directionnelle de la préposition para é fondamentale, en tant qu'elle montre un mouvement d'éloignement, d'un aqui du locuteur vers un autre point de repère, que ce soit un espace physique ou cognitif. Quand il a la valeur spatiale, praí marque un mouvement vers l'espace de l'allocutaire:

(16) • • E nós éramos assim: - Praí hoje não vamos. (12H2A)

[••Et nous disions: aujourd'hui, nous n'y allons pas.]

En ce qui concerne les relations interpersonnelles, ce mouvement vers l'autre prend des dimensions négatives. Le locuteur se met à distance face au contenu et à l'interlocuteur. En (17), praí marque le désaccord et aussi l'irritation envers l'interlocuteur:

(17) ele estava aos berros comigo, eu virei-lhe as costas. $\bullet \bullet$ Fui beber uma cerveja • • e deixei o burro sozinho. • Fala para aí! (12H2A)

[Il était en train de crier sur moi, je lui ai tourné le dos. Je suis allé boire une bière et j'ai laissé cet idiot tout seul. • Ferme ta gueule!]

\subsubsection{Usages approximatifs de praí}

L'usage approximatif de praí est saillant dans notre corpus. Praí, comme aí, peut être un marqueur d'indétermination. En fait, il convie à une interprétation approximative, en tant que il véhicule quelque chose qui n'est pas exactement cela (Vigara Tauste, 1966: 25) ${ }^{19}$.

18 Mihatsch (2009: 101) reprend le schéma de Prince, Bosk et Frader (1982) pour distinguer « Dans le domaine de l'approximation (...) entre les " adaptors », les moyens d'approximation comme espèce de qui servent à indiquer un emploi flexibilisé d'un lexème et les " rounders », les moyens d'approximation numérique, comme environ ».

${ }^{19}$ En espagnol: « no es exactamente eso ». 
Praí, à valeur d'approximateur, est un commentaire métadiscursif (un modalisateur tel que le propose Vion, 2012), par lequel le locuteur se dédouble; en mettant en scène un autre point de vue, un autre énonciateur, il s'éloigne et ne prend pas en charge le point de vue premier. Voyons l'énoncé «Foi praí em dezembro» [c'était p't-être en décembre]. La supression de praí, " Foi em dezembro ", entérine la force épistémique de l'assertion. L'approximateur amoindrit la valeur de vérité de l'énoncé, dont le locuteur ne se porte plus garant. C'est donc une stratégie de déresponsabilisation énonciative.

Nous avons relevé deux dimensions dans les usages approximatifs de praí.

D'abord, sa fonction d'approximateur découle d'un mouvement de reformulation qui brouille l'information. C'est un réajustement du dit qui déresponsabilise le locuteur. Prototypiquement, praí a lieu avec des expressions quantitatives. C'est un cas d'approximation numérique (Mihatsch, 2009: 101). L'exemple ci-dessous est illustratif de la valeur d'approximation qui caractérise tout le segment discursif:

(18) E: 3D está bués de caro agora, não está? Tipo, sete euros ou mais.

I: [...] Está. Cinco. • C Cinco, cinco e tal. E se for 3D é sete e tal praí. • • (03H1B)

[En 3D c'est trop cher, n'est-ce pas? Genre, sept euros ou plus. / Oui. Cinq... Cinq, environ cinq. Et s'il s'agit de 3D c'est sept environ plus ou moins]

En tant que mouvement de reformulation du dire, de son adéquation à l'objet référé, praí accroit l'indétermination et brouille délibérément la dénomination de l'objet:

(19) O ISAVE ou praí qualquer coisa assim. (86M4C)

[ISAVE ou [praí] quelque chose comme ça.]

Les localisations spatio-temporelles sont l'autre domaine de l'usage approximatif de praí:

(20) E: E mas lembras-te da última vez que foste?

I: Lembro-me. Foi/ • • Foi praí • • foi praí em dezembro. (03H1B)

[Et tu te rappelles la dernière fois que tu y es allé?

Oui. C'était/ c'était p't -être c'était p't-être en décembre.]

(21) E: quando é que é o teu próximo jogo? •• Quem? Ronfe. $• •$ E...

I: • É sábado, • • com Ronfe. Ronfe. Acho que é de Famalicão supostamente.

Ou Guimarães. • • Sei que é pra praí, um desses lados. (03H1B)

[Et c'est quand le prochain match? Qui? Ronfe? Et...

C'est samedi, contre Ronfe. Je crois que c'est à Famalicão éventuellement.

Ou à Guimarães. Je sais que c'est par là.] 


\section{Conclusion}

L'analyse de aí et praí soulève des questions complexes mais fort intéressantes du point de vue de la compétence communicative orale, surtout en ce qui concerne le Portugais langue étrangère. Les usages attestés de ces termes mettent en relief la polysémie de ce «petit mot».

Il faut remarquer, à propos de cette série aqui, aí, ali:

- La surdétermination de l'usage des déictiques par le positionnement énonciatif du locuteur. Du déictique au non-déictique il y a un continuum réglé par l'intersubjectivité.

- Le primat de l'accessibilité (de nature cognitive) sur la visibilité de l'objet de discours, ce qui permet de comprendre la valeur de la spatialité déictique comme espace de mémoire.

- Des usages non-déictiques complexifient encore la détermination des valeurs sémantiques et pragmatiques de la série. On peut dire que, à cause de l'éparpillement de ces emplois, ce sont des éléments linguistiques difficiles à cerner. Il faut penser à des catégories souples, i.e., hétérogènes.

En ce qui concerne aí et praí, en particulier, le positionnement énonciatif du locuteur rend compte des usages évaluatifs et approximatifs ici présentés.

Le genre discursif, une interview sociolinguistique à registre colloquial, étaye ces types d'occurrences enregistrées. C'est une interaction à thèmes flottants (ou presque), comme c'est d'usage dans les conversations. Les interviewés parlent de leur vie personnelle, racontent des mémoires.

En fait, les occurrences de notre corpus montrent que l'imprécision du dit et du dire est une stratégie de communication ratifiée par le genre discursif et le registre spontané. Il s'agit de causer, de raconter des récits personnels. La précision narrative est donc accessoire. La dimension évaluative surdétermine les usages d'aí et praí. Et quand on a affaire à un jugement négatif, celui-ci repose sur une sorte de on dit déresponsabilisant.

Il y a donc une incertitude épistémique construite et montrée par ces mécanismes approximatifs et évaluatifs. De plus, ces choix discursifs assurent la déresponsabilisation du locuteur sans atteindre l'efficacité de l'interaction. La précision factuelle n'est pas le premier des objectifs des interlocuteurs.

\section{REFERENCES BIBLIOGRAPHIQUES}

AMOSSY, R., L'argumentation dans le discours, Paris: Nathan, 2000.

BARBERIS J.-M., «Identité, ipséité dans la déixis spatiale: 'ici' et 'là', deux appréhensions concurrentes de l'espace? », in L'Information grammaticale, 77, 1998, pp. 28-32.

BENVENISTE, E., Problèmes de linguistique générale, 2, Paris: Gallimard, 1974.

BÜHLER, K.,Théorie du Langage, Marseille: Agone, 1934 [2009]. 
BUHLER, K.,Teoria del Lenguaje. Trad. Madrid: Alianza Editorial, 1979 [1934].

CUNHA, C. \& CINTRA, L.F., Gramática do português Contemporâneo, Lisboa: Caminho, 1984.

DUCROT, O., Le dire et le dit, Paris: Minuit, 1984.

FONSECA, F. I., Deixis, Tempo e Narração, Porto: Fundação Eng. António de Almeida, 1992.

FONSECA, F. I., Gramática e Pragmática - Estudos de Linguística Geral e de Linguística Aplicada ao Ensino do Português, Porto: Porto Editora, 1994.

FONSECA, F. I., «Deixis e pragmática linguística », in Introdução à Linguística Geral e Portuguesa, org. de I. Hub Faria, E. Ribeiro Pedro, I. Duarte, C. A. M. Gouveia, Lisboa: Editorial Caminho, 1996, pp. 437-445.

FONSECA, F. I., "Deixis e Poesia », in Revista da Faculdade de Letras "Línguas e Literaturas », Porto, XII, 1995, pp. 75-89.

FRASER, B., « Pragmatic competence: the case of hedging », in Kaltenbock, G., Mihatsch W. \& Schneider, S. (org). New Approaches to Hedging, Emerald Group Publishing Limited, 2010, pp. 15- 34.

HALLIDAY, M. \& HASAN, R., Cohesion in English, New York: Longman, 1976.

KLEIBER, G., " Déictiques, embrayeurs, "token-réflexives", symboles indexicaux, etc.: comment les définir? ", in L'information grammaticale no 30., 1986, pp. 3-22.

LANDONE, E., « El alcance interpersonal de los marcadores del discurso en la dinámica conversacional: el ejemplo de la cortesía verbal », in Verba 39, 2012, pp. 301-313.

LYONS, J., Linguistic Semantics. An Introduction, London: CUP, 1995.

MARQUES, M.-A., "O discurso direto em interações orais coloquiais », in M. A. Marques \& X. M. Sanchéz Rei (ed). Novas perspetivas linguísticas no espaço galego-português, Monografia 10. Revista Galega de Filoloxía, Universidade da Corunha, 2015, pp. 89-109.

MIHATSCH, W., "L'approximation entre sens et signification: un tour d'horizon », in Verbeken,D. (ed). Entre sens et signification - Constitution du sens: points de vue sur l'articulation sémantique-pragmatique, Paris: L'Harmatan, 2009, pp. 100-116.

MONDADA, L. \& DUBOIS, D., «Construction des objets de discours et catégorisation: une approche des processus de référentiation", in Revue Tranel (Travaux neuchâtelois de linguistique), vol. 23, 1995, pp. 273-302.

PEREIRA, M., Aspectos Semânticos e Pragmáticos de aqui, aí, ali, cá e lá em Português Europeu, Tese de mestrado. FLUP, 2009.

RABATEL, A., "La part de l'énonciateur dans la construction interactionnelle des points de vue», in Marges linguistiques, 9, 2005, pp.115-36. Disponible en $<$ http://www.margeslinguistiques.com>.

RABATEL, A., "Retour sur les relations entre locuteurs et énonciateurs. Des voix et des points de vue », in Linguistiques 32, 2010, pp. 357-373, <halshs-00504918>.

RAPOSO, E., « Advérbio e sintagma adverbial », in Raposo et al. (org.) Gramática da Língua Portuguesa, Lisboa: Fundação Calouste Gulbenkian, 2013, pp. 1569-1684.

TEIXEIRA, J., «De cá para lá e de aqui para aí: rede de valores semânticos dos marcadores espaciais cá / lá (acolá) e aqui / aí / ali », in Rio-Torto, Graça Maria; Figueiredo, 0. M. \& Silva, F. (coord.): Estudos em Homenagem ao Professor Doutor Mário Vilela, vol. I, Porto: Faculdade de Letras da Universidade do Porto, 2005, pp. 449-460. 
VASQUEZ CUESTA, P. Luz, M. A., Gramática da Língua Portuguesa, Lisboa: Edições 70, 1971. VIGARA TAUSTE, A. M., " Español coloquial: expresión del sentido por aproximación », in T. Kotschi, W. Oesterreicher y K. Zimmermann (eds.), El español hablado y la cultura oral en España e Hispanoamérica, Frankfurt-Madrid: Vervuert-Iberoamericana, 1996, pp. 15-44.

VION, R., « Modalités, modalisations, interaction et dialogisme », in Brès, J. P., Haillet, S. Mellet, H. Nølke, L. Rosier (orgs). Dialogisme, polyphonie: approches linguistiques. Bruxelas: De Boeck Duculot, 2005, pp.143-156.

\section{Annexe}

\section{Conventions de transcriptions:}

nous avons utilisé les signes de ponctuation traditionnels des règles de l'écriture (.,?,!,:;); mots superposés: (aaa); signes paraverbaux: ((aaa));

fragments incompréhensibles ((aaa));

interruption (aaa/)

discours direct $(:-)$

pause longue $(\bullet \bullet \bullet)$,

pause courte $(\bullet \bullet)$

intervieweure (E:);

interviewé (I:).

Note: fragmentes en italiques: structure analysées. 